\title{
Wind Powered Switched Reluctance Generator for Rural Properties and Small Communities.
}

\author{
Fleury, A. ${ }^{1,2}$, Andrade, D. A. ${ }^{3}$, Silveira A. W. F. V. ${ }^{3}$, Ribeiro, P. H. F. ${ }^{1}$, Melo, F. S. L. F. ${ }^{1}$, Migliorini, L. A. G. ${ }^{1}$, \\ Dias, D. N. ${ }^{1}$, Oliveira, J. I. ${ }^{1}$ \\ ${ }^{1}$ Electrical Machines and Drives Laboratory \\ Pontifical Catholic University of Goias \\ University Square, 1440 - Goiania (Brazil) \\ ${ }^{2}$ Physics Laboratory \\ State University of Goiás \\ Road 153 - Anápolis (Brazil) \\ ${ }^{3}$ Electrical Drives Laboratory \\ Federal University of Uberlandia \\ Saint Monica Campus - Uberlandia (Brazil) \\ Phone number:+55 629611 5775, e-mails: afleury@terra.com.br, darizon@ufu.br, gutofleury@gmail.com, \\ faleiroribeiro@hotmail.com,fredfsm@hotmail.com, luisandregm@hotmail.com,dannogueiradias@hotmail.com
}

\begin{abstract}
The subject matter of this paper is the Wind Powered Switched Reluctance Generator - WPSRG for complementary use in rural properties and in small communities. It deals with the power grid connected operation of the wind powered machine. The excitation source of the WPSRG is a rectified single phase AC line. A mathematical model for the SRG is presented and confirmed. This model takes into account the saturation and the mutual inductances effects. A low cost power converter topology connects the wind power to the power grid in DC avoiding frequency and voltage adjustment problems. The converter is used as a part of a really new arrangement that allows the grid to fully feed the load without any switching when the WPSRG is not running. Simulations and experimental results are presented. These investigations will be helpful to broaden the comprehension of WPSRG systems in grid connection.
\end{abstract}

\section{Keywords}

Clean energy, Electric energy generation, Switched reluctance generator, Wind power.

\section{Introduction}

Nowadays the global consumption of energy presents a stable growth tendency [1]. Production, distribution and use of the energy must be as efficient as the available technology allows. Scattered loads like rural properties and small communities are frequently supplied through Single Wire Earth Return - SWER line type. The voltage is low and the length is great. Maximum load demand close to 1MVA or 2MVA are quite frequent. Small KVA per kilometer ratios recurrently cause economic losses. The voltage in these lines presents strong oscillations depending on its loading.

The use of load tap changing transformers is expensive and inconvenient in many cases and this power supply arrangement implies the use of single phase devices by the end users, increasing their energy costs. The installation of standard generating units closer to these scattered loads uses to be also not profitable. Thus the implementation of diffuse enterprises using renewable energy are now encouraged as a contemporary strategic alternative. But when an enterprise of this sort is isolated there are problems of continuity and when it is grid connected there are problems of voltage and frequency adjustments.

At the present time wind power has experienced great development and its market share is increasingly growing as the cost of wind power overcomes the competitiveness barrier [1].

Wind powered generators must operate efficiently under variable speed conditions. Although the efforts are getting it from conventional machines, a devoted special machine is welcome. The switched reluctance machine represents one of the simplest types of electrical machines and the Switched Reluctance Generator - SRG is a potential device for variable speed power generation. Investigations conducted to explore this new option showed that it is promising due to the new advances in power electronics and in micro processing. The SRG is reliable, efficient and presents some specific characteristics that match the needs of wind power.

This paper discusses the use of a Wind Powered Switched Reluctance Generator - WPSRG to supply rural properties and small communities side by side with the grid through a devoted converter topology. With it the power from the WPSRG is coupled to the power of the grid in DC. Thus synchronism problems are avoided. A PWM inverter can adjust a three phase voltage and frequency at the output of the converter. The proposed 
arrangement allows the grid to fully feed the load when the WPSRG is not running [2]. A SRG prototype was built and tested under variable speed and variable load conditions. Saturation and mutual inductances effects were considered. The results are presented here. They confirm that the SRG works well under those conditions and that the WPSRG can be successfully used as a complementary source of electric power.

\section{Wind Energy Potential}

The instantaneous power density $\left(P_{i}\right)$ that can be derived from a wind flow at a certain high over the ground level is given by:

$$
P_{i}=\frac{1}{2} \rho_{i} v_{i}^{3} C_{i}
$$

where $v_{i}$ is the affluent wind speed in $\mathrm{m} / \mathrm{s}$ at a certain height, $\rho_{i}$ is the air density in $\mathrm{kg} / \mathrm{m}^{3}$ at height and $C_{i}$ is the turbine efficiency. For a three-blade low-speed turbine the efficiency of the rotor less than 0.4. Thus the following expression for density of power in such turbines is regarded here as technologically acceptable:

$$
P_{i}=0.15 \rho_{i} v_{i}^{3}
$$

In order to make an inventory of wind energy potential at a site, the density of the average wind kinetic power can be estimated through the following modified algorithm:

$$
P=\frac{0.15}{8760 n} \sum_{i=1}^{8760 n} \rho_{i} v_{i}^{3}
$$

where $P\left(W / m^{2}\right)$ is the average annual wind power density which can be derived from the site and $n$ is the number of measurements taken in each hour. It must be emphasized that the air density varies with the altitude, the temperature and the pressure at a given site. Regarding these aspects, equation (3), evaluated with experimental measurements, is propitious for an assessment of wind power potential available in a certain site.

Equation (3) shows that the integration which allows calculating the average power that can be derived from a site depends on the cube of the wind speed at each measurement performed. Thus, if the average value of $\rho_{i}$ is measured, the result of the integration will depend on the root mean cube speed. It is a consensual appreciation that the wind speed in a certain site follows the Weibull probability distribution function like equation [3]:

$$
p\left(v_{i}\right)=\left(\frac{k}{c}\right)\left(\frac{v_{i}}{c}\right)^{(k-1)} e^{-\left(\frac{v_{i}}{c}\right)^{k}}
$$

where $p\left(v_{i}\right)$ is the fraction of time where wind speed is between $v_{i}$ and $v_{i}+\Delta v_{i}$, divided by $\Delta v_{i}, c$ is a scale parameter and $k$ is a shape parameter. Generally $p\left(v_{i}\right)$ is expressed in hours per year per $\mathrm{m} / \mathrm{s}$. On most places c varies from 5 to $10 \mathrm{~m} / \mathrm{s}$ and $\mathrm{k}$ varies between 1.5 and 2.5 [3].
Thus the root mean cube speed $\bar{v}_{c}$ is given by the following equation:

$$
\overline{v_{c}}=\sqrt[3]{\frac{1}{8760 n} \int_{0}^{\infty} p\left(v_{i}\right) \times v_{i}^{3} d v_{i}}
$$

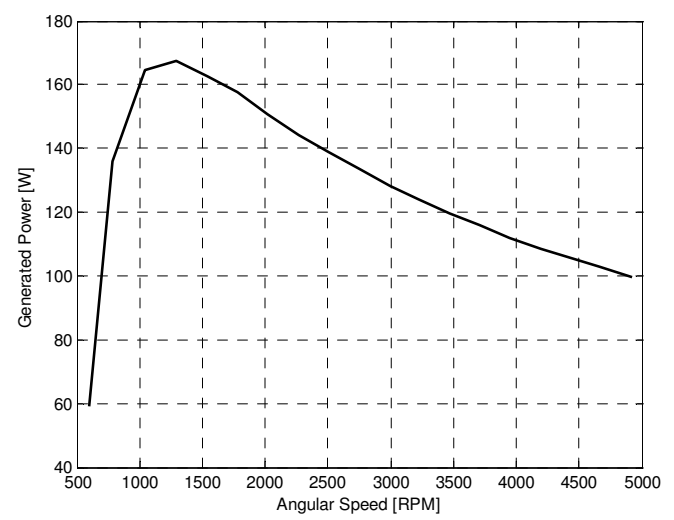

Fig. 2. Generated power as a function of angular speed for a Switched Reluctance Generator prototype.

With this, the average power density at a site is:

$$
P=0.15 \bar{\rho}^{3}
$$

where $\bar{\rho}$ is the average value for the air density in that site. Both equations (3) and (6) depend on long time local measurements.

A quite simple analysis conducted by equation (4) shows that the wind speed is low most of the time and the wind turbine rotor speed trend is to be a mirror of the wind speed. The generator which is consonant with this feature must reach its point of highest generation still at low speeds and must be able to keep its generating capacity while the wind speed increases.

Fig. 2 depicts the power generated by a constructed prototype of switched reluctance generator with 6 poles in the stator and 4 poles in the rotor, excited at $29 \mathrm{~V}$, when its speed goes from $500 \mathrm{rpm}$ to $5000 \mathrm{rpm}$. This experimental result shows that the SRG fits into the characteristic of angular speeds expected from the wind turbines which work in places of lower average speed of the wind.

\section{Using the Wind Power}

Technological developments are increasingly broadening the utilization of wind turbines. At this time the blades are bigger, the towers are higher and the rated power has increased substantially. New control techniques are being used and the operational speed range is becoming wider. The gearbox is now dispensable and the nacelle became lighter. There has been an appreciable noise reduction. While these developments are going on, the costs are going down. Furthermore, a wind farm is highly modular and the required physical area is small, which decreases initial investment costs.

However, isolated use of wind power is inadvisable because of the unreliability of the primary source. So, it is necessary to share the service to consumers with other 
power sources. The use of diesel-driven generators associated to one or few isolated wind turbines is usual, but this solution is not good as it implies to install extra capacity to meet $100 \%$ of the demand with diesel-driven generation and afterwards to restrict its use making the end price of each $\mathrm{kWh}$ produced higher than it could be. The use of batteries for energy storage is an expensive solution and can be applied only when the loads are quite small. The combination of complementary renewable sources increases the reliability, but still keeps a considerable risk of deficit due to unexpected weather or seasonal conditions. Taking it all that into consideration, a solution that uses the single phase power grid connected transmission lines side by side with wind turbines without energy interruption would be attractive. The losses along the lines will decrease and the wind power use will be safe. A WPSRG allows it as will be shown in the following sections [4].

\section{The Switched Reluctance Generator}

Fig. 3 shows parts of the SRG prototype tested. The doubly salient poles structure and the phase coils concentrated only in the stator poles are remarkable features of this machine [5]. There is a minimal thermal loss in the rotor [6]. This machine is easy to construct and to maintain. Its most commonly quoted advantages are: absence of permanent magnets [2], low manufacturing costs, reliability, robustness, efficiency [7] and a wide operational speed range. Current, voltage and torque ripples that can be controlled by the power electronics used for its driving. Some quoted applications are: aeronautics, as a starter-generator for cars and the wind power applications.

Switched reluctance machines can work as motors or as generators just by changing their switching angles. For the configuration proposed in this work, the SRG has a mechanical input and an electrical input. Its output is the electrical power supplied to the load. The machine presents two different electric circuit configurations per phase: one for the excitation period and other for the generation period. The converter topologies that drive a SRG must consider its singularities.

In a SRG, when a pole of the rotor is aligned with the excited pole of the stator, there is a state of stable equilibrium. Thus, there is a natural tendency to align the rotor and the stator active poles, in order to maximize de inductance of that phase. When a mechanical agent forces the rotor to leave the stable equilibrium position, the electromagnetic torque produced results in a electromotive force that increases the applied voltage. In this way the machine generates electric power.

The electrical equation for a phase of the $6 \times 4$ SRG is:

$$
\begin{aligned}
& v_{a}=R_{a} i_{a}+\frac{d \theta}{d t}\left(L_{a a} \frac{d i_{a}}{d \theta}+L_{a b} \frac{d i_{b}}{d \theta}+L_{a c} \frac{d i_{c}}{d \theta}\right)+ \\
& +\frac{d \theta}{d t}\left(\frac{\partial L_{a a}}{\partial \theta} i_{a}+\frac{\partial L_{a b}}{\partial \theta} i_{b}+\frac{\partial L_{a c}}{\partial \theta} i_{c}\right)
\end{aligned}
$$

where $v_{a}$ is the phase $a$ winding voltage; $i_{a}, i_{b}$ and $i_{c}$ are the phases currents; $R_{a}$ is the resistance of the phase $a$ winding; $L_{a a}$ is the self inductance of phase $a ; L_{a b}$ is the mutual inductances among phases $a$ and $b ; L_{a c}$ is the mutual inductances among phases $a$ and $c ; \theta$ is the rotor angular position and the so the rotor angular speed is:

$$
\omega=\frac{\mathrm{d} \theta}{\mathrm{dt}}
$$

The induced electromotive force is given by:

$$
e=\frac{d \theta}{d t}\left(\frac{\partial L_{a a}}{\partial \theta} i_{a}+\frac{\partial L_{a b}}{\partial \theta} i_{b}+\frac{\partial L_{a c}}{\partial \theta} i_{c}\right)
$$

The stator winding is fed in DC. When the back electromotive force is positive electric power is converted to mechanical power and the machine works as a motor. But when the back electromotive force is negative it increases the current converting mechanical power into electrical power and the machine acts as a generator.

The dynamic mechanical equation for the SRG is given by (10). It is to be noted that the electromagnetic torque $\mathrm{C}_{\mathrm{emag}}$ comes as a negative quantity, so acting against the rotor mechanical speed.

$$
\mathrm{C}_{\mathrm{m}}+\mathrm{C}_{\mathrm{emag}}-\mathrm{J} \frac{\mathrm{d} \omega}{\mathrm{dt}}-\mathrm{D} \cdot \omega=0
$$

where $C_{m}$ and $C_{e m a g}$ are the mechanical torque and the electromagnetic torque respectively, $\mathrm{J}$ is the inertia and $\mathrm{D}$ is the coefficient of friction.

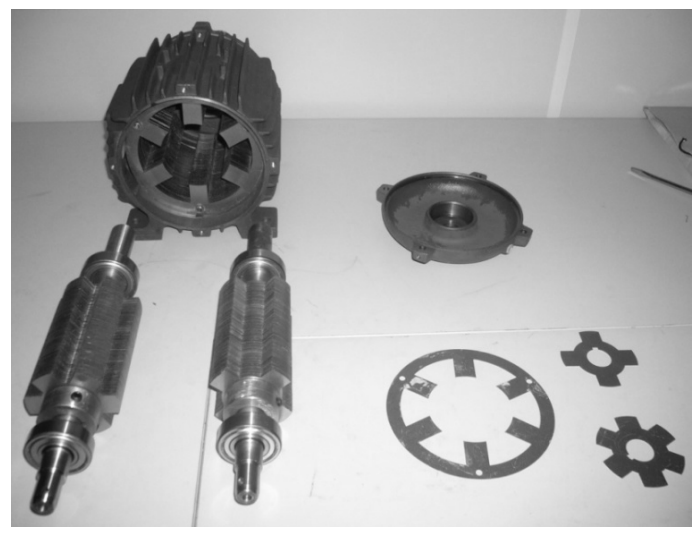

Fig. 3. Parts of a prototype of Switched Reluctance Generator.

The co-energy $\left(W^{c o}\right)$ of a phase of this machine is given by:

$$
\mathrm{W}^{\mathrm{co}}=\int_{0}^{\mathrm{i}} \lambda \mathrm{di}
$$

where $\lambda$ is the magnetic flux in that phase.

And the corresponding electromagnetic torque for a three phase SRG is given by:

$$
C_{\text {emag }}=\sum_{x=1}^{3} \frac{\partial W_{x}^{c o}}{\partial \theta}
$$

The mathematical model of the SRG regarding a three phase prototype is shown below:

$$
\left[\begin{array}{c}
v_{a} \\
v_{b} \\
v_{c} \\
C_{m} \\
0
\end{array}\right]=\left[\begin{array}{ccccc}
R_{a} & \omega \frac{\partial L_{a b}}{\partial \theta} & \omega \frac{\partial L_{a c}}{\partial \theta} & 0 & 0 \\
\omega \frac{\partial L_{b a}}{\partial \theta} & R_{b} & \omega \frac{\partial L_{b c}}{\partial \theta} & 0 & 0 \\
\omega \frac{\partial L_{c a}}{\partial \theta} & \omega \frac{\partial L_{c b}}{\partial \theta} & R_{3} & 0 & 0 \\
-\frac{\partial W_{a}^{c o}}{\partial \theta} & -\frac{\partial W_{b}^{c o}}{\partial \theta} & -\frac{\partial W_{c}^{c o}}{\partial \theta} & D & 0 \\
0 & 0 & 0 & -1 & 0
\end{array}\right]\left[\begin{array}{c}
i_{a} \\
i_{b} \\
i_{3} \\
\omega \\
\theta
\end{array}\right]+
$$




$$
\left[\begin{array}{ccccc}
L_{a a} & L_{a b} & L_{a c} & 0 & i_{a} \frac{\partial L_{a a}}{\partial \theta} \\
L_{b a} & L_{b b} & L_{b c} & 0 & i_{b} \frac{\partial L_{b b}}{\partial \theta} \\
L_{c a} & L_{c b} & L_{c c} & 0 & i_{c} \frac{\partial L_{c c}}{\partial \theta} \\
0 & 0 & 0 & J & 0 \\
0 & 0 & 0 & 0 & 1
\end{array}\right]\left[\begin{array}{c}
\cdot \\
i_{a} \\
i_{b} \\
\dot{i_{c}} \\
\dot{\omega} \\
\dot{\theta}
\end{array}\right]
$$

If the matrices above are designated $[V],[R],[I],[L]$ and $[\dot{I}]$ exactly in the order they appear in (13), the matrix of states for the SRG has the form:

$$
\dot{I I}]=[L]^{-1}[V]-[L]^{-1}[R][I]
$$

\section{Computing Model}

Fig. 4 shows a block diagram for the SRG wind power systems proposed in this article. All the electrical power delivered to the load comes from both the mono phase source and the SRG [8].

To get a mathematical model for the three phase $6 \times 4$ SRG prototype constructed, experimental measurements of the self and the mutual inductances were done for many rotor positions $(\theta)$, also considering different current values for each one of them. These measurements resulted in a large data bank of the functions $L(\theta, i)$. Using this data bank, programs for polynomial interpolation were constructed to evaluate the inductance of a phase as a function of its current and the instantaneous rotor position. In this way also the effects of the saturation are considered in the simulation results. A new electronic converter topology, here named Series Converter (SC), was used to drive the machine. It requires only a diode and a switch per phase. The SC topology showed in Fig. 5 is devoted to the SRG. It allows supplying the load even when the wind turbine is still.

The power coming from an AC source is rectified to both excite the generator windings and to feed the load. As in a conventional SRM operation, the phases are energized sequentially, one at a time. The excitation period of a phase begins when its switch is turned on and starts to conduct. At this moment the corresponding diode is not conducting and the phase winding generates a positive back electromotive force. The generating period starts when the controlled switch is turned off. The phase current is deviated to the load through the diode and the phase windings generate a negative back electromotive force, which adds to the supply voltage (eq. (7)). Fig. 6 shows the process described above.

The mathematical model presented in equations (13) and (14) was checked using a computing program whose inputs are the phase voltages and the mechanical torque. The outputs are the phase currents, the angular speed and the rotor position. Each new set of values for the phase voltages and the torque is used as a new input in the program to evaluate the next state. The program uses dynamic values that result from the relationship among the components of the converter circuit and from the relations among them and the $\mathrm{AC}$ source, the rectifier bridge, the machine and the load. Only the information of the rotor position is necessary to control the gate signals. This simulation strategy allows checking the converter behavior under different circumstances like torque, speed or load changes.

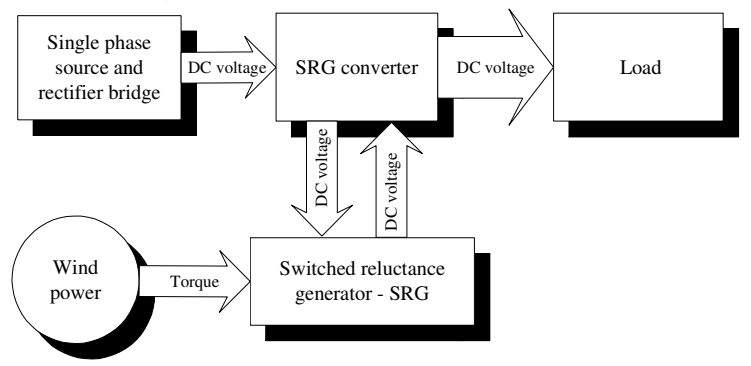

Fig. 4. Block diagram of SRG wind power system.

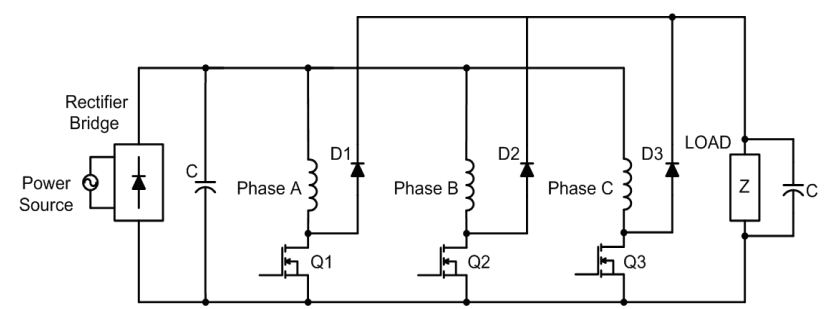

Fig. 5. Electrical scheme of Series Converter (SC).

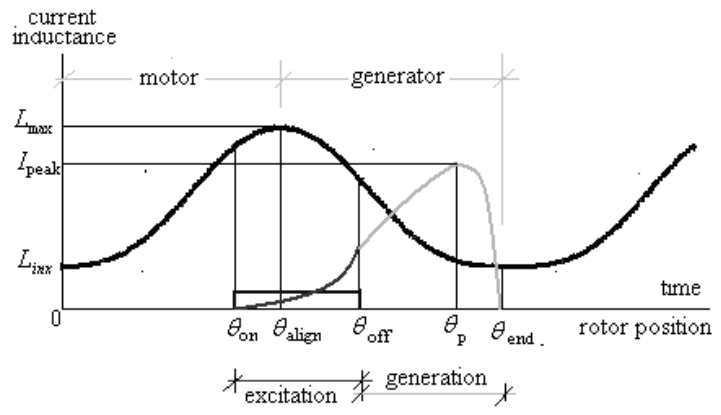

Fig. 6. Excitation and generation periods.

\section{Simulation Results}

The simulations were done using data from a small prototype of SRG. Its parameters and dimensions are given in Table I.

Each phase winding has 60 turns of copper wire AWG 15. The driving strategy states that each phase is fired during 30 degrees and just one phase is fired at time.

The SRG was simulated under different conditions. Simulation results at a typical speed of $1300 \mathrm{rpm}$, using the SC topology, will be presented. And there are others to present in a full text.

Once these simulation take into account the mutual inductance effect these effects will be discussed a few. Also de capability of the SC converter to supply the load using both the generated power and the grid power will be presented and discussed in the full paper. 
TABLE I

Characteristics of SRG used

\begin{tabular}{|c|c|c|}
\hline Parameter & Value & Units \\
\hline Stator Diameter & 140 & $\mathrm{~mm}$ \\
\hline Rotor Diameter & 70 & $\mathrm{~mm}$ \\
\hline Stack Length & 107 & $\mathrm{~mm}$ \\
\hline Air Gap Length & 0.4 & $\mathrm{~mm}$ \\
\hline Stator Teeth Width & 19 & $\mathrm{~mm}$ \\
\hline Rotor Teeth Width & 20 & $\mathrm{~mm}$ \\
\hline Stator Slot & 22.5 & $\mathrm{~mm}$ \\
\hline Rotor Slot & 11.7 & $\mathrm{~mm}$ \\
\hline Stator Yoke & 12 & $\mathrm{~mm}$ \\
\hline Rotor Yoke & 12.4 & $\mathrm{~mm}$ \\
\hline Shaft Diameter & 22 & $\mathrm{~mm}$ \\
\hline Number of turns per phase & 50 & turns/phase \\
\hline Inertia & 0.0028 & $\mathrm{~kg} \cdot \mathrm{m}^{2}$ \\
\hline Coefficient of Friction & 0.026 & N.m.s \\
\hline Inductance (Aligned Position) & 36 & $\mathrm{mH}$ \\
\hline Inductance (Unaligned Position) & 3 & $\mathrm{mH}$ \\
\hline Conducting Angle & 30 & degrees \\
\hline
\end{tabular}

In the simulations the conduction angle of the phase switch began at 4.7 degrees before the peak of the inductance of this phase. The excitation period of this phase ended at 25.3 degrees after that peak. From this point on, the phase transfers power to the capacitor and to the load until the rotor reaches the minimum inductance position at around 45 degrees. The dynamic for the next phase is always the same.

The SRG generates power in separated pulses, and this points the need of an end capacitor to smooth the load voltage.

Running at $1300 \mathrm{rpm}$ under stable conditions, the system absorbs power from the AC source and from the mechanical source. The converter supplies power from these two sources to the load. The electric resistances of the windings, the switches, and the diodes were considered in the simulations. There are measured losses mainly located in the windings. According to the results, the SRG presented good efficiency under regular operation. Long-time simulations were done and the model of the machine showed stable behavior running with different fixed load and different constant speeds.

\section{Experimental Results}

Tests were carried out to confirm the mathematical model and the simulation results. A variable voltage AC source was used to control the performance of the entire system. A rectifier bridge with a capacitor of $5 \mathrm{mF}$ was used to provide the DC input power from the grid. The converter was assembled with MOSFET and diodes. The resistive load was of $10.8 \Omega$ and a $20 \mathrm{mF}$ capacitor was used to smooth the load voltage.

In the first test the SRG was driven at the speed of 900rpm. Fig. 7 shows the waveforms after the rectifier, which corresponds to DC link supply used to excite the SRG and to supply the load. The measurements were $31.6 \mathrm{~V}$ and $9.35 \mathrm{~A}$ to the link DC, with an input power supply of $295,5 \mathrm{~W}$. For this test the desired output power supply to the load was set up in $380 \mathrm{~W}$.
The output results obtained as consequence of that input and of the generation process are shown in Fig. 8. The values measured in the output were $60.5 \mathrm{~V}, 6.3 \mathrm{~A}$ and $381 \mathrm{~W}$. Considering the $295.5 \mathrm{~W}$ coming from the DC input, the SRG is delivering more $85.5 \mathrm{~W}$ to the load. Therefore, the machine generated $85.5 \mathrm{~W}$ and all the losses for the generating process.

In Fig. 9 the voltage at a single winding is compared to that obtained by simulation with the computing model under the same operational conditions.

In the second test the SRG was kept still and AC supply voltage applied into the diode rectifier. The waveforms before the rectifier in this new test were measured as $65.2 \mathrm{~V}$ and 6A. So the input power supply rated $391.2 \mathrm{~W}$, considering the unitary power factor of the diode bridge rectifier.

The waveforms of the voltage and current feeding the load at the output, were close to those of Fig.8. Their measurements were $63.3 \mathrm{~V}$ and $6 \mathrm{~A}$ respectively, supplying the load with $379.8 \mathrm{~W}$. As the SRG was not running, the output results obtained were only due to the process of passing through the windings and the converter. Considering the $391.2 \mathrm{~W}$ at the DC input and that the SRG was not giving power to the load, leading to joule losses of $11.4 \mathrm{~W}(3 \%)$.
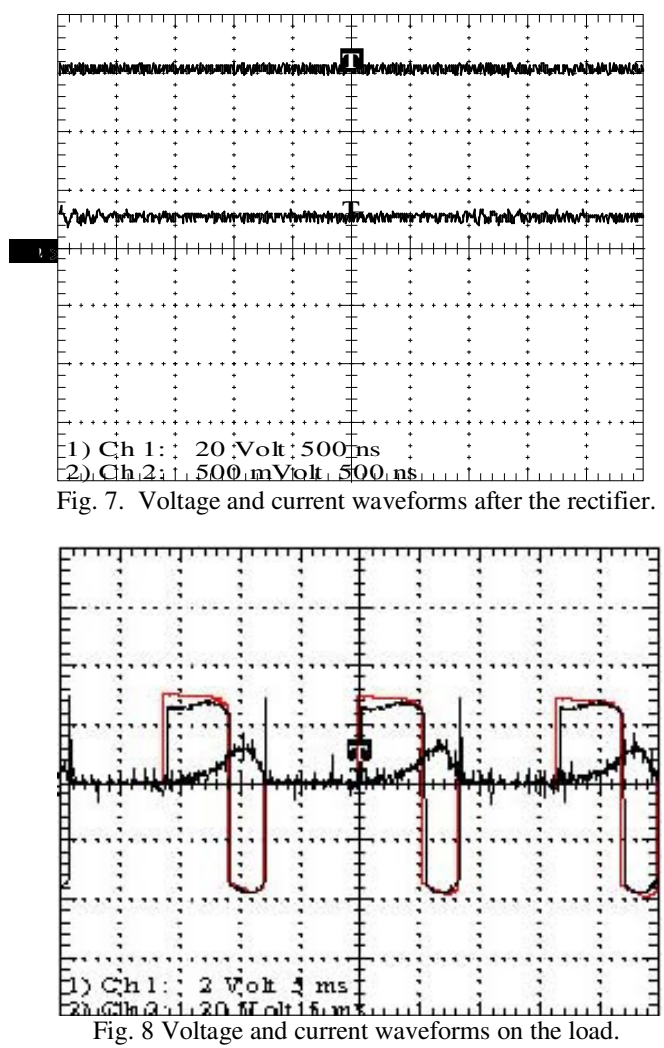

The results shows that for each excitation voltage, the generated power quickly reaches its maximum value when the machine spins at a little more than $1200 \mathrm{rpm}$. It was also verified that the speed which corresponds to the maximum power generated depends on the excitation voltage. This find indicates that a complete control strategy based only in the excitation voltage can be used to drive a WPSRG according to the load needs.

The load voltage also could be stabilized through adjustment of the excitation. These tests confirm that the 
controlled switching of the rectified voltage at the input of the converter definitely can be used to help stabilize the voltage over the load.

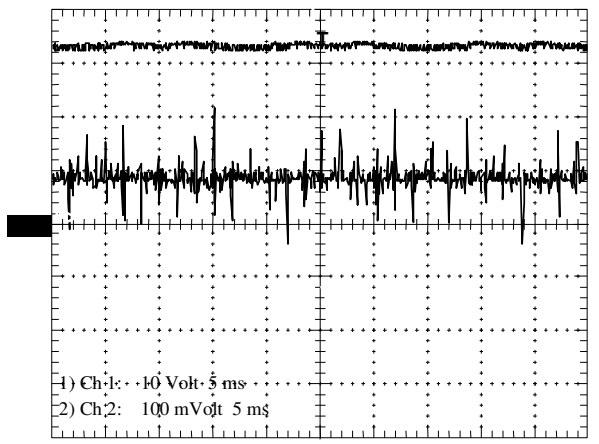

Fig. 9. Voltage and current waveforms over a winding.

Furthermore, to control a WPSRG it must be taken into consideration that the load is a part of the electrical circuit during the generating period. And so the load voltage depends on the load itself. Tests with the small prototype constructed were conducted to evaluate the load changes effects. Its results are presented here.

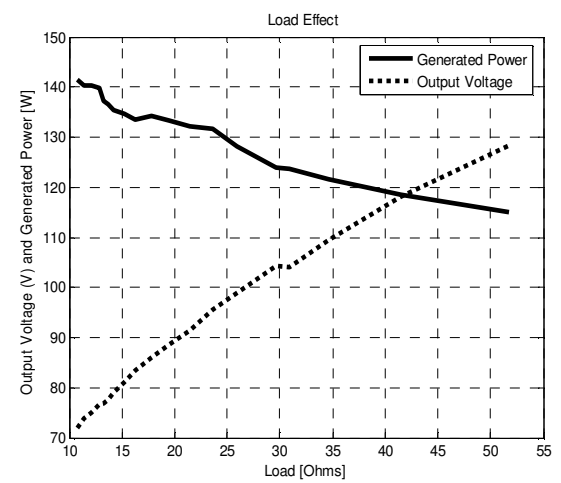

Fig. 10. Experimental results regarding the load effect over the average output voltage and the average power generated with the SRG prototype.

For these tests the excitation voltage was kept at $35 \mathrm{~V}$ and the speed fixed in $1200 \mathrm{rpm}$ while the load resistance was changed step by step from $10.8^{\prime} \Omega$ to $51.7 \Omega$. Results are shown in Fig. 10: when the load decreases, the average voltage increases as expected, and, in the opposite direction, the generated power decreases. Due to the small size of the prototype the voltage rise is large. Although as the load decreased $378 \%$, from $10.8 \Omega$ to $51.7 \Omega$, its voltage increased at around $78 \%$, from $72 \mathrm{~V}$ to $128 \mathrm{~V}$. This means that without any voltage control the small prototype changed its output voltage 4.8 times less than the load change.

The SRG prototype did not present any harmful or unexpected behavior with changes in its rotor speed or in its excitation voltage or in the load.

The experimental results presented here point that a feasible control strategy of the excitation voltage can easily adjust and stabilize the output voltage as desired.

Side by side with the results presented and announced, in the full text a study on the mutual inductance influence on the global SRG performance was investigated and will be presented.

\section{CONCLUSIONS}

Rural properties and small communities are sparse loads.
They are fed by single phase transmission lines with poor voltage regulation. Regular solutions for this problem use to be anti economic. To install WPSRG close to these loads is a contemporary strategic alternative. The switched reluctance machine works well as a generator. Its power profile depending on the rotation speed matches the classical wind speed probability distribution. The mathematical model presented and confirmed by the tests conduced depends on the mutual inductances. A cheap converter topology allows the grid to fully feed the load without any switching when the SRG is still. This converter interfaces the SRG and the power grid in a simple way. An arrangement with the specific converter topology was assembled. A prototype was constructed and tested. Surfaces were plotted to show the experimental results. It can be seen there that the power supplied to the load presents a single maximum for each value of the rotor speed under the same excitation voltage. Also the output voltage dependence on the rotor speed and on the excitation voltage is depicted. Finally, the output voltage and power depending only on the load magnitude is analyzed. All the output parameters can be fully controlled through the excitation voltage control. The tests conduced show the operational behavior a SRG prototype as it was not presented until now. These data will be helpful to control a WPSRG system connected to the power grid.

\section{Acknowledgement}

The authors thanks the Pontifical Catholic University of Goias, the State University of Goias and the Federal University of Uberlandia for their support given to this work.

\section{References}

[1] F. Blaabjerg, F. Iov, "Wind power - A power source now enabled by power electronics," in 9th Brazilian Power Electronics Conference Records, september, 30 to October, 3, 2007, Blumenau, Brazil.

[2] [3] A. Fleury, D. A. Andrade, F. S. Silva, J. L. Domingos, "Switched Reluctance Generators Behavior under Different Conditions", in IEEE International Symposium on Industrial Electronics, ISIE'07 records, 2007.

[3] M. R. Patel, "Wind and solar power systems" in CRC Press, New York, 1999, 350 pp..

[4] A. Fleury, D. A. Andrade, "Switched reluctance Generator for Complementary Wind Power Generation in Grid Connection" in International Electric Machines and Drives Conference IEMDC 2007, Antalya. IEMDC'07, 2007. v. CD-ROM.

[5] R. Cardenas, R. Peña, M. Perez, G. Asher, J. Clare, O. Wheeler, "Control system for grid generation of a switched reluctance generator driven by a variable speed wind turbine," in Industrial Electronic Society, 2004. IECON 2004. $3^{\text {th }}$ Annual Conference of IEEE, Vol. 2, 2-6 nov. 2004, pp. 1879-1884.

[6] D. A. Andrade, R. S. Costa, R. S. Teixeira, A. Fleury, "Energy Efficiency for Fractional Power Loads," Industry Applications Magazine, Vol. 12, No 6, November|December 2006, pp. 12-20.

[7] M. A. MUELLER, "Design and performance of a $20 \mathrm{~kW}, 100$ $\mathrm{rpm}$, switched reluctance generator for a direct drive wind energy converter," in IEEE International Conference on Electrical Machines and Drives, 15-18 May 2005, pp. 56-63.

[8] Ribeiro, F.S. L., Cabral, L. G., Fleury, A., "A Switched Reluctance Generator Behavior under Variable Speed and Variable Excitation", in ICREP 2008. 